\title{
Nursing Activities at Health Surveys and Health Checkups during the Early Period of Operation of the Hiroshima ABCC-Oral History Study Based on Narratives of Three Japanese Nurses
}

\author{
Saori Funaki, Mizue Shiromaru \\ Department of Nursing, School of Health Sciences, Sapporo Medical University, Sapporo, Japan \\ Email: ootake@sapmed.ac.jp
}

How to cite this paper: Funaki, S. and Shiromaru, M. (2018) Nursing Activities at Health Surveys and Health Checkups during the Early Period of Operation of the Hiroshima ABCC-Oral History Study Based on Narratives of Three Japanese Nurses. Open Journal of Nursing, 8, 69-77. https://doi.org/10.4236/ojn.2018.81006

Received: December 25, 2017

Accepted: January 23, 2018

Published: January 26, 2018

Copyright $\odot 2018$ by authors and Scientific Research Publishing Inc. This work is licensed under the Creative Commons Attribution International License (CC BY 4.0).

http://creativecommons.org/licenses/by/4.0/

\begin{abstract}
Using an oral history approach, this study analyzed the narratives obtained from semi-structured interviews administered to three Japanese women who worked as nurses for the Hiroshima Atomic Bomb Casualty Commission (ABCC). The analysis of the data in the narratives showed details of the health surveys and health checkups conducted during the early period of operation of ABCC. During the early period of operation of ABCC some survivors showed negative behaviors toward the health surveys and health checkups at the ABCC. However, it can be inferred that dedicated nursing played an important role in alleviating the stress and emotional issues of survivors at the health surveys and health checkups. The findings of the study could be beneficial to research into nursing activities for patients exposed to radiation at the present day.
\end{abstract}

\section{Keywords}

Hiroshima, ABCC, Atomic Bombs, Health Care of the Radiation-Exposed, Narrative (Oral History Approach)

\section{Introduction}

\subsection{Background}

After the atom bomb attacks on Hiroshima and Nagasaki in 1945, USA established an Atomic Bomb Casualty Commission (ABCC) during the postwar oc- 
cupation to conduct long-term investigations into the effects of radiation on survivors of the atomic bombs [1]. This study uses an oral history approach, and collected data on details of the nursing activities at the health surveys and health checkups conducted by the ABCC by interviewing three Japanese nurses who worked at the Hiroshima ABCC during this period. Even after the detonation of the atomic bombs, today there are still patients exposed to radiation due to the development of nuclear weapons and energy generation including nuclear tests and accidents like those at the Chernobyl and Fukushima Daiichi nuclear power plants, showing the necessity to provide nursing support for those exposed to radiation. The focus of this study is the unusual and extraordinary experiences related to the nursing activities at Hiroshima ABCC, and we believe the study offers ideas that will be useful for continuous nursing support in the health care of those exposed to radiation. Further, the experience of the nurses who were engaged in the long-term nursing support for the survivors of the atomic bombs in the unique environment provided by the $\mathrm{ABCC}$, established during the occupation period is valuable and worth keeping on record.

\subsection{Objectives}

The study aims to determine the nursing activities at the health surveys investigating details of health hazards due to the atomic bombs and at the health checkups for individual subjects during the early period of operation of the Hiroshima $\mathrm{ABCC}$ through the narratives of nurses working at the ABCC.

\subsection{Significance of Study}

The focus of this study is on unusual and extraordinary experiences related to the nursing activities at Hiroshima ABCC, however we believe the findings of this study will be useful in the continuing nursing support in health care today for those exposed to radiation for any reason.

\section{Methods}

\subsection{Participants}

Participants in the study reported here are Japanese, former nurses who worked for the Hiroshima ABCC from 1947 to 1975, when the ABCC was dissolved. During the early period of operation of the Hiroshima ABCC six American nurses and twenty-eight Japanese nurses worked for the ABCC [2], and this number had changed to two American nurses and thirty-four Japanese nurses in June, 1954 [3]. This study was conducted upon receiving written consent for participation from Ms. A, Ms. B, and Ms. C who all worked for the Hiroshima ABCC.

\subsection{Procedures to Recruit Study Participant}

To obtain cooperation for the survey, we made a request conforming to the following procedures: We 1) explained an outline of the survey and ethical consid- 
erations to the director of the Radiation Effects Research Foundation (RERF), and obtained cooperation; 2) requested the head of the medical department to recommend prospective participants, obtained consent for participation in the study from two former nurses, and further conducted sampling from a network of former nurses recommended by the RERF to introduce prospective participants, resulting in obtaining consent from one former nurse; and 3) explained the ethical considerations and data collection methods to the former nurses of the ABCC who had consented to the participation both orally and in writing, and also obtained consent to access public personal information.

\subsection{Interview Guide}

Prior to the interview we mailed the participants a document that explained the semi-structured interviews and a questionnaire, and the author of the present study conducted interviews at a conference room of the Hiroshima office of the RERF. The interviews took one to two hours for each participant. The questions described in the questionnaire sent to the participants in advance are shown below:

1) main duties at the ABCC, 2) details of the work assignment of the personnel at the $A B C C$, their interactions and relationships in the work, 3) how the surveys investigating details of health hazards due to the atomic bombs were conducted at the ABCC, 4) how health checkups to determine health hazards due to the atomic bombs were conducted at the ABCC, and 5) which matters the nurses placed value on in relation to the survivors of the atomic bombs.

\subsection{Data Collection Period}

Data were collected from October, 2016 to the end of January, 2017.

\subsection{Data Collection and Analysis}

This study used an oral history method [4]. The analysis is carried out in the following process.

1) We transcribed the recorded narratives from the digital voice recorder, carefully and accurately, noted everything down in writing, and this forms the testimony, 2) we read through the testimony repeatedly, 3) asked the participants to confirm whether the testimony is correct and whether there is any faulty information, 4) we integrated the experiences reported in the testimony with the analysis of the printed sources considering the study purpose, and conducted further interviews as this was thought necessary, and 5) finally examined whether the data analysis and interpretation reflected the records truthfully through checking by different members of the team as well as with supervision by experts in oral history and history of science, to improve the validity of the analysis.

\subsection{Ethical Considerations}

This study was conducted with approval from the Ethics Committee of Sapporo Medical University (Approval No.: 28-2-33). We explained the details of the 
study including the study objectives, methods, significance, participation as voluntary, the right to discontinue the participation at will, and that interviews may be discontinued if an interviewer determines that any of the participants harbor feelings of anxiety, stress, physical pain, or other kinds of impediment due to the interview. We also explained the necessity to access the personal information such as personal histories and backgrounds of the participants because in the oral history approach the narrative of a person will become historical evidence and because oral history is an approach that expresses the personality of the individual clearly, and we ensured that we had obtained consent to participate in the interviews.

\section{Results}

\subsection{Backgrounds of the Participants}

A) Work period at the ABCC, B) Process to become employed by the ABCC

1) Ms. A, Female

a) 1949 to 1966, b) introduced by a nurse working for the ABCC who were in the same year in nursing school

2) Ms. B, Female

a) 1951 to 1987 (ABCC and RERF), b) Recommended by a grandmother living in Hiroshima, and took a recruitment examination for the employment

3) Ms. C, Female

a) 1964 to 1996 (ABCC and RERF), b) Applied for the post of nurse at the ABCC published in a classified advertisement of the Chugoku Newspaper.

\subsection{Nursing Activities at Home Visits for the Genetics Surveys}

As a part of the health surveys, the ABCC started the Major Genetics Study in March of 1948 by opening an office leased by the Hiroshima Red Cross Hospital. The narrative of Ms. A, who had been engaged in home visits provided details of home visits to infants, showing the main role of participants in the genetics study. The following is from the narrative of Ms. A:

"(In the Genetics Study Department) When I visited homes with a physician, I assisted the physician in the examination of newborn babies."

"Before the home visit, conductors (contact persons) made an appointment to visit the homes of patients."

"A driver took a physician and a nurse using a US Army jeep to and from the home, referring to address records for residences of patients. In those days, it was hard to find the homes of patients driving on bumpy, and dusty roads without car navigation."

"Entering the room, the physician asked the mother about the baby's condition while the nurse spread out a brown sheet of paper, and put out a stethoscope and tongue depressor. Then, the physician examined the patient (the baby), measuring body parts. If any abnormality was observed, the nurse drew blood from the mother." 
"The Genetics Study Department was operated provisionally using a part of the Hiroshima Red Cross Hospital, but remained here for a period of time without moving the office even after the completion of a new facility in $\mathrm{Hi}-$ jiyama in 1950."

From the narrative of Ms. A, it was determined that the main role of nurses was to assist physicians when examining patients at home visits, and that the home visits for the genetics study were conducted by three persons, a Japanese physician, a nurse, and the driver using a US Army jeep. The genetics study continued in an office in the Hiroshima Red Cross Hospital even after a new facility in Hijiyama was completed in 1950.

\subsection{Nursing Activities at Health Checkups in the Facility of the ABCC}

As described above, the dedicated facility for the ABCC was completed in November of 1950 and some of the examinations of the health surveys were conducted here in the departments of internal medicine, pediatrics, genetics study, dentistry, ophthalmology, gynecology, and surgery [1]. The narratives of the participants showed that at the health checkups physicians used imported medical equipment, and that one nurse attended each patient who visited the ABCC. Details of the health checkups by each of the departments visited and the reactions of the radiation exposed survivors who were the subjects of the health checkups were also provided. The following is from the narratives of Ms. A, Ms. B, and Ms. C.

Narrative of Ms. A:

"Nurses had to be at work by 8 o'clock to prepare for the day, and the examination of patients started at 9."

"I escorted patients and assisted physicians in examining the patients."

"When patients visited the $A B C C$, health checkups were conducted in the following manner: patients were led to the examination room from the reception desk. In a corner of the examination room, we asked the patients to change clothes and put on a gown. Woman patients were also wearing a drape, made of white cotton cloth, with an opening at the center under the gown. For patients who did not wear the drape in the examination, nurses covered the abdomen and back with a hand towel. In the gynecology department, nurses wrapped sheets around both legs of patients. This was to prevent unnecessary exposure. The main role of nurses was to assist physicians in this manner. Then, they went to the X-ray room in another building. After that patients returned to the examination room, the checkup finished with providing urine and blood samples, and measurements of height, weight (recorded in inches and pounds), temperature, and blood pressure."

"I think that the pediatric examination focused mainly on measurements 
of the body. The ABCC conducted fundus examinations in the department of ophthalmology, imaging of capillaries in the dentistry, and tumor examinations in the gynecology, surgery, and dermatology departments."

"Because we, both physicians and nurses dedicated ourselves to the duties to serve the patients, we had no time to talk personally with the patients. We never heard patients including pediatric patients complain or express dissatisfaction with the proceedings, rather we strongly believed that they were satisfied."

Narrative of Ms. B:

"I met patients who visited the ABCC at the entrance. They came to the $\mathrm{ABCC}$ because they were persuaded by the conductor (contact person) to do so. One nurse attended one patient from the gowning at the examinations till the consultation by the physician, and was present at the send-off of the patient at the entrance."

"A friend of mine who still continues to take the follow-up examination every two years told me she was called a guinea pig at the early examinations. She thought the visit was troublesome when she was young. But as she was getting older, she felt differently. She felt secure by having the checkups at the ABCC. Everyone is kind and polite to her. It's perfect. She expressed her gratitude to the ABCC."

Narrative of Ms. C:

"All medical equipment was imported. Everything including urinary bottles and lavatory bowls were large. The bed sizes were also larger than those of Japan, and wash basins were heavy, too."

These health checkups at the ABCC were conducted with control groups of people who had not been exposed to radiation and the control groups were comprised of the same number of checkups as the survivors of the atomic bombs. From the narrative of Ms. C, it was found that the ABCC paid special attention to the tests and attitudes towards the survivors of the atomic bombs without telling the nurses that the people who came to have health checkups were survivors of the atomic bombs, to ensure the conduct of fair and unbiased health checkups. The following is from the narrative of Ms. C:

"I didn't know at all (who was a survivor of the atomic bomb and who was not). There was no indication of this even on the chart (medial record). I found out about it only when patients told me about what had happened to them at the atomic bombings. What being exposed to the radiation was like, we didn't ask them questions like that."

"I never asked the patients of their backgrounds. This was because it was a rule not to discriminate among the patients at the checkups." 


\section{Discussion}

\subsection{Nursing Activities at the Health Surveys Investigating Details of Health Hazards Due to the Atomic Bombs and at the Health Checkups for Individual Subjects}

\subsubsection{Nursing Activities at Home Visits for the Genetics Study}

Relevant to the home visits for infants by the ABCC, the narrative of Ms. A showed the difficulties in conducting home visits in post-atomic Hiroshima. Lindee reported one factor that made the visits difficult was to find the place to visit because there were no street signs in Hiroshima and Nagasaki after the end of the war [5]. The narrative of Ms. A also suggests the difficulty in traveling by jeep because of the poor road conditions and because housing was chaotic just after the end of the war.

The narrative of Ms. A also suggests that three persons, a Japanese physician, a nurse, and a driver made the home visits for the genetics study using US Army jeeps. It was also found that physicians carried basic instruments such as a stethoscope and measure in a bag. The main role of nurses at the home visits was to assist physicians with examining the infants, including arranging the medical instruments for the convenience of the physicians and collecting blood samples when necessary. James V. Neel, who planned and implemented the genetics study, noted that workers in one of the positions that played an important role in the genetics study were the Japanese nurses who made the home visits [6]. This implies that these Japanese nurses played a significant role in the genetics study, which was a large scale home visit survey in post-atomic Hiroshima.

\subsubsection{Nursing Activities at Health Checkups in the Facility of the ABCC}

At the ABCC, the health checkups were conducted by the various departments there, including the major pediatric research program started in March, 1949 in Hiroshima, and the major adult medical investigations project started in August, 1950 [1]. From the narratives of the three nurses, detailed procedures of and items examined at the health checkups were identified. According to the narrative of Ms. C, it was found that the medical equipment used in the ABCC was imported, implying that the nurses had difficulty in using and dealing with the equipment because the sizes and weights were different from that which was made in Japan. According to the narrative of Ms. A, the main role of nurses at the health checkups was to assist physicians, and one nurse attended one patient throughout a health checkup paying careful attention to prevent unwarranted exposure for the female patients. These suggest that attentive nursing was provided to the patients. From the narrative of Ms. B, it became clear that the attitudes and concerns of survivors of the atomic bombs toward the checkups by the ABCC changed over time. According to Ms. B, one patient felt that she was treated like a guinea pig at the checkups by the ABCC, but later she felt secure and supported by the checkups and expressed that everybody was kind and supportive. Ms. A stated that physicians and nurses dedicated themselves to their duties towards the patients. As some studies have shown negative opinions 
towards the research programs of the ABCC [7] [8] and as the narratives of participants here show, it seems that many survivors of the atomic bombs had negative feelings toward the health surveys and health checkups of the ABCC at first. However, such negative feelings toward the $\mathrm{ABCC}$ changed to become positive with the passage of time. This may be because, as described above, the nurses at the $\mathrm{ABCC}$ provided devoted attentive nursing to the patients. Positive opinions of the nursing activities at the ABCC as expressed by James V. Neel [6], a geneticist of $\mathrm{ABCC}$ during the early period of the ABCC, and Robert W. Miller, a pediatrician, suggest that the quality of the nursing provided was good [9]. Therefore, it may be inferred that the nurses at the ABCC played important roles in alleviating the stress of the survivors of the atomic bombs at the health surveys and health checkups by paying attention to the emotions of the survivors.

The health checkups of the health surveys by the ABCC targeted both the survivors of the atomic bombs and non-exposure-survivors. From the narrative of Ms. C it was learned that nurses were not given information about exposure to radiation although they interviewed patients about medical history, family background, and main complaints. The nurses also paid close attentions to attitudes towards the survivors of the atomic bombs and performed their duties carefully to insure that problems with this did not affect the test results. Ms. C stated that this is because the ABCC was a research institute and because they had to treat both the survivors of the atomic bombs and non-exposure-survivors equally at the health checkups.

\subsubsection{Study Limitations and Issues to Be Addressed in the Future}

The study analyzed the nursing activities focusing on the health surveys investigating details of health hazards due to the atomic bombs and the health checkups of individual subjects during the early period of operation of the Hiroshima ABCC. Since the ABCC also conducted activities in Nagasaki, Japan, future investigation needs to include the nursing activities in the Nagasaki ABCC in the same period.

\section{Conclusion}

This study aimed to determine the nursing activities at the health surveys investigating details of health hazards due to the atomic bombs, and at the health checkups for individual subjects conducted by the ABCC during the early period of the Hiroshima $A B C C$ using an oral history approach, and analyzed the narratives of Japanese former nurses who worked for the ABCC. The analysis showed that the ABCC had a mission to collect precise data as a research institute, and nurses had provided nursing care while paying attention to the attitudes shown towards the survivors of the atomic bomb. At the ABCC imported medical equipment was used, and the main role of the nurses was to assist physicians at the health surveys and health checkups. However, it was suggested that nurses at the ABCC provided dedicated nursing by one nurse attending one patient and paying attention to prevent feelings of embarrassment of the female survivors. 
The findings suggest that dedicated nursing played an important role in alleviating the negative emotional issues of survivors at the health surveys and health checkups which were harbored by some survivors during the early period of operation of the ABCC.

\section{Acknowledgements}

We wish to express our gratitude to Ms. A, Ms. B, and Ms. C for sharing their valuable experience for the purpose of this study. We are also grateful to Dr. Kazunori Kodama and Ms. Akemi Wakamoto at the Radiation Effects Research Foundation for providing valuable information and advice related to the research activities of ABCC. This research received no specific grant from any funding agency in the public, commercial, or not-for-profit sectors. The authors declare that they have no conflicts of interest to report.

\section{References}

[1] Atomic Bomb Casualty Commission of National Institute of Public Health, Ed. (1978) 1947-1975 ABCC and National Institute of Public Health Joint Report. Atomic Bomb Casualty Commission, Hiroshima, 222-223. (In Japanese)

[2] Atomic Bomb Casualty Commission, Quarterly Report for Period 1 January 1950 thru 31 March 1950, Nursing, 31. March-1950 (Owned by Hiroshima Radiation Effects Research Foundation).

[3] Atomic Bomb Casualty Commission, Semi Annual Report, 1 January 1954 through 30 June 1954, Nursing, 30. June-1954 (Owned by Hiroshima Radiation Effects Research Foundation).

[4] Nakamura, M. (2008) Digging out the Memory of Showa-Extreme Situations in Okinawa, Manshu, Hiroshima, and Nagasaki. Shougakukan, Tokyo, 13. (In Japanese)

[5] Lindee, M.S. (1994) Suffering Made Real: American Science and the Survivors at Hiroshima. The University of Chicago Press, Chicago, 57-115.

[6] Neel, J.V. (1990) The Very Early Years of the ABCC Genetics Program, 1946-1951. RERF Update, 2, 6-9.

[7] Nakagawa, Y. (1986) A Historical Reexamination on the Investigations of Atomic Radiation Effects in Hiroshima and Nagasaki: Underestimations of Acute Radiation Effects. Journal of History of Science, Phase II 25, 20-33. (In Japanese)

[8] Sugihara, Y. (1967) Issues around ABCC. Journal of Japanese Scientists, 2, 29-33. (In Japanese)

[9] Miller, R.W. (1993) Miller's Memories of ABCC-RERF, 1953-1990, Part 1. RERF Update, 5, 7-9. 